\title{
Abstract: 4D Template Library Generation for Real-Time Tracking on 2D Cine MRI
}

\author{
Luis Paredes ${ }^{1}$, Paul Mercea ${ }^{2,3}$, Hendrik Teske ${ }^{2,3}$, Rolf Bendl ${ }^{2,3,4}$, \\ Kristina Giske ${ }^{2,3}$, Ignacio Espinoza ${ }^{1}$ \\ ${ }^{1}$ Instituto de Física, Pontificia Universidad Catolica de Chile, Santiago, Chile \\ ${ }^{2}$ Division of Medical Physics in Radiation Oncology, DKFZ Heidelberg \\ ${ }^{3}$ National Center for Radiation Research in Oncology (NCRO), Heidelberg Institute \\ for Radiation Oncology (HIRO) \\ ${ }^{4}$ Faculty of Computer Science, Heilbronn University
}

lfparedes@uc.cl

In radiotherapy, fast image-based tracking of tumor motion during radiation is conventionally achieved with template matching approaches on x-ray based projection scans with limited soft-tissue contrast. Emerging MR guided radiotherapy (MRgRT) devices allow for ionization-free imaging during treatment with superior soft tissue contrast. Currently, real-time imaging with MR is only possible for single slice acquisition (2D cine MRI). In this type of acquisition, breathing-induced tumor motion may change the appearance of the target in the scanned plane. This out-of-plane target motion may affect the accuracy of tracking algorithms based on template matching. In this work, an advanced 4D multiple template library approach was developed in order to enable 3D target localization on 2D cine MRI. The image data used in this work consists of: i) training data of 84.7 seconds of parallel sequential cine MRI (4.5Hz, 11 slices, 256x256px size, $1.56 \mathrm{~mm}$ pixel spacing, $4.5 \mathrm{~mm}$ slice thickness, 35 repetitions) capturing the target throughout its motion and ii) tracking data of 200 single slices of cine MRI simulating the real-time data provided by an MRgRT device. A retrospective self-navigated phase-based $4 \mathrm{D}$ sorting algorithm was developed for $4 \mathrm{DMRI}$ reconstruction of the training data from which the phase dependent multiple templates were extracted. These templates were incorporated in a real time multiple-template matching based tracking algorithm which was executed on the tracking data. For evaluation purposes, an automatic target contouring algorithm was developed and manually generated reference data was used. The 4D self-navigated reconstruction algorithm was able to generate 4DMRIs (256x256x31px, isotropic voxel size) from the training data for up to 10 phases of the respiratory cycle. The tracking results reproduced quasi-elliptic 3D target trajectories comparable (mean error $0.76 \pm 0.76 \mathrm{~mm}$ and max error $3.8 \mathrm{~mm}$ in through-plane direction) to the ones captured with a device-side 4DMRI sequence (acquisition time $>450$ seconds). In comparison to the reference data, the mean tracking error in coronal orientation was $0.62 \pm 0.91 \mathrm{~mm}$ in superiorinferior and $0.45 \pm 0.80 \mathrm{~mm}$ in left-right direction. Out-of-plane motion effects in cine MRI can lead to reduced precision of dose administration in beam tracking. With our approach, accurate estimation of the 4D trajectory of the target volume is realized and out-of-plane motion can be assessed. 